\title{
CLINICAL UTILITY OF EDACS-ADP IN PATIENTS ADMITTED WITH CHEST PAIN TO AN EMERGENCY DEPARTMENT
}

\author{
Ilker Akbas ${ }^{1} \oplus$, Zeynep Cakir ${ }^{\circledR}$, Abdullah Osman Kocak² ${ }^{2}$, Alpaslan Ünlü3, \\ Nazim Onur Can ${ }^{4}$, Mert Vural ${ }^{2}$, Muhammed Zübeyr Köse ${ }^{2}$ \\ 'Department of Emergency Medicine, Bingöl State Hospital, Bingöl, Turkey \\ ${ }^{2}$ Department of Emergency Medicine, Faculty of Medicine, University of Ataturk, Erzurum, Turkey \\ ${ }^{3}$ Department of Emergency Medicine, School of Medicine, Recep Tayyip Erdoğan University, Rize, Turkey \\ ${ }^{4}$ Department of Emergency Medicine, Erzurum Regional Training and Research Hospital, Erzurum, Turkey
}

\begin{abstract}
INTRODUCTION: Acute coronary syndrome (ACS) is a common cause of mortality and morbidity. An ACS diagnosis can be made with electrocardiogram (ECG) and cardiac markers. However, despite medical advances, $2-5 \%$ of ACS patients are undiagnosed and discharged from emergency departments (EDs) because clinicians often find it difficult not only to diagnose and treat high-risk patients but also to define nonemergency diseases or safely discharge healthy patients. Risk stratification can be prevented, and inappropriate diagnosis and treatment protocols can be identified. The ED Assessment of Chest Pain Score-Accelerated Diagnostic Protocol (EDACS-ADP) scoring system, developed to identify patients with chest pain but at low risk for a major adverse cardiac event (MACE), is the first score based on clinical data from emergency medicine.

AIM: This study investigates the usability of EDACS-ADP in Turkey.
\end{abstract}

MATERIAL AND METHODS: This is a prospective observational study of 392 patients. The primary outcome was a major adverse cardiovascular event (MACE) within thirty days.

RESULTS: A total of 116 MACEs occurred in $65(16,6 \%)$ patients during a one-month follow-up. The sensitivity, specificity, positive predictive value (PPV), negative predictive value (NPV), positive likelihood ratio (+ LR), and negative likelihood ratio (-LR) values of the EDACS-ADP score for the evaluation of 30-day MACE rate in patients who admitted with chest pain for two months were as follows: $96.9 \%, 64.5 \%, 35.2 \%, 99.1 \%,+$ LR: 2.73, and-LR: 0.05 .

CONCLUSIONS: Most of these patients were classified by the EDACS-ADP as low risk and suitable for discharge. The 30-day MACE rate of development was significantly low $(0.9 \%)$ and acceptable in patients grouped as low risk.

KEY WORDS: chest pain, chest pain score, EDACS, ADP

Disaster Emerg Med J 2021; 6(1): 33-40

\section{INTRODUCTION}

Chest pain is one of the most common complaints worldwide, requiring rapid, accurate diagnosis and evaluation. In $3 \%$ to $6 \%$ of all emergency admissions, the only complaint is chest pain [1]. In the United States (U.S.), emergency services garner

ADDRESS FOR CORRESPONDENCE:

Ilker Akbas, Department of Emergency Medicine, Bingöl State Hospital, Bingöl Turkey e-mail: akbasilker@gmail.com 
8-10 million visits per year due to chest pain, which is the second most common cause of admission and the number of annual ED visits is increasing [2-4]. Chest pain may be caused by non-life-threatening factors such as myalgia or by severe causes such as aortic dissection or pulmonary embolism. Although an ED evaluation usually focuses on serious pathologies, the last diagnoses in most patients are non-life-threatening causes. Acute coronary syndrome (ACS), pulmonary embolism, and aortic dissection are diagnosed in $12,5 \%$ of patients presenting to the ED with chest pain or discharged from the hospital after evaluation [5]. ACS, which is most commonly associated with chest pain, is a common cause of mortality and morbidity today. The diagnosis of ACS can be made by using ECG and cardiac markers; however, normal ECG and cardiac data do not mean the disease does not exist. Despite all the advances in medicine, $2-5 \%$ of patients with acute myocardial infarction (MI) are discharged from the ED without being diagnosed. These "missed MI" cases account for $40 \%$ of emergency service malpractice cases $[6,7]$.

For these reasons, when patients who have chest pain are evaluated in the ED, clinicians often find it difficult not only to diagnose and treat high-risk patients but also to identify non-emergency diseases or safely discharge those who have no disease. [8]. In patients who have chest pain, risk stratification can be prevented, and unnecessary diagnosis and treatment protocols can be identified. Also, lowrisk patients can be discharged from the ED quickly, reducing overcrowding and health costs $[8,9]$. In contrast, the correct diagnosis of high-risk patients prevents premature discharge and allows these patients to be observed for longer times with all the facilities. For this reason, most current publications and guidelines recommend the use of a risk scoring system for patients presenting to the ED with chest pain $[8,10]$. There are many scoring systems for evaluation of patients with chest pain or ACS, but most of them are designed to predict the potential for adverse events in patients undergoing intensive care or to distinguish patients at high risk from those with diagnosed ACS. Very few scoring systems are used for identifying low- to moderate-risk patients who may be discharged among those who present to the ED with chest pain $[8,9]$.

The ED Assessment of Chest Pain Score-Accelerated Diagnostic Protocol (EDACS-ADP) scoring system, which was developed to identify chest pain patients with a low risk for MACE, is the first system based on clinical data from emergency medicine $[9,11]$. The EDACS-ADP was created in 2014 by Than et al. [12], using information from patients who participated in the ADAPT study, and later validated in Australia and New Zealand, where it was developed. Researchers focused on two goals in developing the scoring system. The first goal was to develop a clinically reasonable score to predict the short-term risk of MACEs in adults presenting to the ED with a complaint of angina. The second aim was to accurately identify the low-risk group for MACE and to safely and quickly discharge them. Therefore, researchers developed the EDACS using a troponin assay and an ECG at the second hour and an accelerated diagnostic protocol (ADP) feature [12]. The EDACS-ADP can correctly classify more than half of those admitted to the ED with chest pain (51\%) as low risk for MACE, allowing for early discharge. The rate of MACE development at the end of the thirty days after early discharge was $0.3 \%[8,9]$. Since its development, the EDACS-ADP has been validated by many prospective and retrospective studies in different countries. This study aims to investigate the usability of the EDACS-ADP scoring system as a risk stratification tool in Turkey.

\section{Study design and setting}

This is a prospective observational study carried out on patients coming to the Ataturk University Hospital Emergency Medicine Clinic between 2/1/2017 and 3/31/2017. The diagnosis, treatment, and management of the patients included in the study progressed completely in accordance with the chest pain and ACS procedures in the hospital, and the discharge decision was made following these same procedures. The study was conducted independently of these protocols, and only the second-hour troponin value was measured from patients evaluated and recommended by a physician.

\section{Study population}

The Ataturk University Hospital is a third-step public hospital. Located in Erzurum in Turkey it is the largest hospital in this province and operates an advanced diagnosis and treatment center for twelve provinces in its immediate vicinity. Approximately 120,000 patients are admitted to its ED annually, either by ambulance (112) or referral from hospitals 
in the surrounding provinces and districts. The hospital has a 24-hour coronary angiography laboratory with a team that is on-call during working hours and experienced in percutaneous coronary intervention $(\mathrm{PCl})$. The dedicated biochemical laboratory for ED patients provides uninterrupted service throughout the day.

Patients over 18 years of age who were admitted to the ED with chest pain for more than five minutes and who read the consent form and agreed to participate were included in the study. The study included the troponin measurements taken in the ED. In the presence of any of the following criteria, the patient was excluded from the study: i. patients under the age of 18 years, ii. those who did not agree to participate in the study, iii. patients diagnosed with a certain reason for chest pain after initial examination without the need for further imaging and laboratory examination (e.g., thoracic wall pathologies, costochondritis, fibromyalgia, mastalgia, etc.), iv. patients with noncoronary pathologies as the cause of chest pain after imaging and laboratory tests (pneumonia, pulmonary embolism, pneumothorax, pericarditis, myocarditis, aortic dissection, GIS-induced pathologies), v. patients presenting with trauma-induced chest pain, vi. patients who did not think a cardiac test and follow-up necessary, vii. patients diagnosed with ST-segment elevation myocardial infraction (STEMI), viii. patients who were involved in the study before, ix. patients who did not have access to medical records, or who could not be reached after 30 days of follow-up, or who died due to cardiovascular reasons during the follow-up period, and $\mathrm{x}$. patients developing cardiac arrest during evaluation in the ED.

\section{Data collection}

To record the patients' information, a study form was prepared in advance. This form was filled out by the study physician, who was not the patients' primary physician, by using the face-to-face interview method after evaluating patients in the emergency room, before the discharge or admission decision was made. Thus, the researchers were prevented from bias, and the study did not hinder the medical evaluation. A one-hour coordination and training meeting was held with the physicians who were likely to fill out the study form. Clinical symptoms and medical history were obtained by asking the patient directly, regardless of the medical records of the patient. If the patient was not sure of the answer (e.g., a history of hypertension), the answer was marked as "No."

\section{Calculation of the EDACS-ADP}

More than three risk factors were identified for those aged 18 to 50 years: family history of premature coronary artery disease (CAD), dyslipidemia, diabetes, hypertension, current smoker, or history of seven heart diseases. Each of the variables has different negative or positive values according to its weight, and the total score is obtained by summing the values of the variables. The score of the two variables is negative, that is, when the scores of these precursors are collected, they are mathematically extracted from the total score. The result gives a patient score between -10 and 34. EDACS values above 16 indicate a high risk of MACE $[10,12,13]$. Table 1 shows the EDACS-ADP and the scores obtained from the variables [12]. If the EDACS is $<16$ for a patient, if there is no new ischemic change in the ECG, and if both troponin values in the second hour are negative, this patient is considered low risk. Patients in this group may be discharged early in the outpatient period [12]. If the EDACS is $>16$, or the ECG shows new ischemic changes, or if any of the troponin values are positive at the time of arrival or by the second hour, these patients are considered middle or high risk. If the initial troponin values are not taken, a delayed troponin test is performed [10]. For the EDACS, 16 is chosen as a cutoff because it maintains a sensitivity of $99 \%$ for MACE, while its specificity is maximum [10].

The patients' EDACS scores were calculated after discharge. Following the methodology for the EDACS-ADP derivation, a variable was considered normal or negative when calculating points during the study. In this way, while the study's reliability was maintained, the sensitivity was kept at the lowest obtained value, and, if the missing data were considered abnormal or positive, it was assumed that the sensitivity and specificity of the study would decrease [13].

\section{ECG evaluation}

When a patient was admitted to the ED with chest pain, an ECG was performed within the first five minutes according to protocol. A copy of this ECG was kept with the study form and these results were evaluated in terms of new ischemia by two emergency physicians. If there was a difference between the physicians' ECG interpretations, a third blinded 


\begin{tabular}{|c|c|}
\hline Clinical features & Point \\
\hline $\begin{array}{l}\text { Age } \\
18-45 \\
46-50 \\
51-55 \\
56-60 \\
61-65 \\
66-70 \\
71-75 \\
76-80 \\
81-85 \\
86+\end{array}$ & $\begin{array}{l}+2 \\
+4 \\
+6 \\
+8 \\
+10 \\
+12 \\
+14 \\
+16 \\
+18 \\
+20\end{array}$ \\
\hline Male gender & +6 \\
\hline $\begin{array}{l}\text { Between } 18 \text { and } 50 \text { years old } \\
\text { i. Known coronary artery disease* } \\
\text { ii. More than three risk factors** }\end{array}$ & +4 \\
\hline Sweating (diaphoresis) & +3 \\
\hline $\begin{array}{l}\text { Pain radiates to the arm or } \\
\text { shoulder }\end{array}$ & +5 \\
\hline $\begin{array}{l}\text { Pain occurred or worsened by } \\
\text { inspiration }\end{array}$ & -4 \\
\hline Pain reproduced by palpation & -6 \\
\hline Low-risk patient & $\begin{array}{l}\text { All criteria must be } \\
\text { provided } \\
\text { 1. EDACS }<16 \\
\text { 2. No new ischemia in } \\
\text { the ECG } \\
\text { 3. Negative series of } \\
\text { troponin }\end{array}$ \\
\hline Risky patient & $\begin{array}{l}\text { The existence of any } \\
\text { criteria is sufficient } \\
\text { 1. EDACS }>16 \\
\text { 2. The presence of new } \\
\text { ischemia in the ECG } \\
\text { 3. Positive series of } \\
\text { troponin }\end{array}$ \\
\hline
\end{tabular}

ADP — accelerated diagnostic protocol; ECG — electrocardiography; EDACS — Emergency Department Assessment of Chest Pain Score-Accelerated Diagnostic Protocol * Known coronary artery disease: a history of previous acute myocardial infarction, past coronary artery bypass graft surgery, previous coronary percutaneous intervention. ** Risk factors: family history of premature CAD, dyslipidemia, diabetes, hypertension, current smoker.

physician made the final decision. The ECGs of the patients included in the study were considered to have new ischemia in the presence of at least one of the following changes: i. at least 0.05 millivolt $(\mathrm{mV})$ ST-segment depression in two or more adjacent leads and T wave inversion at a depth of ii. $0.1 \mathrm{mV}$ or more (negativity), iii. a $\mathrm{Q}$ wave presence longer than 30 milliseconds and greater than $0.1 \mathrm{mV}, \geq 0.2 \mathrm{mV}$ for leads V1, V2, V3 from iv. J point, an ST-segment height in at least two adjacent leads with a size of $\geq 0.1 \mathrm{mV}$, or $\mathrm{v}$. a new left bundle branch block.
These changes were considered as old changes if present in the patient's old ECG or medical records. If there were no records to prove that the changes were old, they were evaluated as new ischemic changes. Data from the validation study of Flaws et al. [13] were taken as examples for this evaluation.

\section{Evaluation of troponin results}

The first troponin assay was done in the emergency room, and a second-hour troponin assay was performed on patients that the doctor was considering following. The serum troponin measurements were performed using a DXI 800 Beckman Coulter (U.S.) instrument. A troponin measurement of $0.04 \mathrm{mi}-$ crogram / L, which is the 99th percentile and above the reference limit, was accepted as the clinical threshold in determining myocardial injury during the study period. The EDACS-ADP was classified as high risk if there were values above this limit in the troponin measurements at the first or second.

\section{Key outcome measures}

The primary outcome was MACE. MACE development information was investigated by phone call and/or by examining the hospital records thirty days after the patient's discharge. If any of the following conditions developed over the thirty days, MACE formation was considered positive: i. acute MI (STEMI and NSTEMI), ii. emergency revascularization requirement, iii. cardiovascular death, iv. cardiogenic shock, or v. highgrade atrioventricular block or ventricular arrhythmia requiring access. Coronary artery bypass graft (CABG), stenting of the coronary artery, and other PCls were considered as the necessity for emergency revascularization. MACE development was evaluated as positive in a patient who was admitted with chest pain after discharge from the hospital, not after discharge or hospitalization. For this reason, it was assumed that all NSTEMI patients developed MACE directly.

\section{Statistical analysis}

The SPSS 20.0 statistical software package was used for statistical analysis. Values were given with frequency, percentage, mean, and standard deviation. Pearson's Chi-square and Fisher's exact test were used to interpret the data. A Kolmogorov-Smirnov distribution test was used to examine the normal distribution, and a Mann-Whitney $U$ test was used to compare the parameters between groups. Sensitivity, specificity, positive predictive value (PPV), and negative predictive value (NPV) were 
calculated according to the cutoff value. Results were evaluated at a 95\% confidence interval and a significance level of $p<0.05$.

\section{RESULTS}

A total of 18,597 patients were admitted to the ED. Chest pain was the most common reason for 1004 (5.4\%) of these patients. Of these 1004 patients, 612 were excluded from the study due to various reasons, 206 patients refused to participate in the study, and 107 patients with chest pain complaints were discharged from the ED without any investigation after the first examination and were not included in the study. In this group of patients, the diagnosis was mostly chest wall pathologies. Since 54 patients were diagnosed with STEMI after the first ECG because of trauma-related chest pain, 146 patients were excluded due to the lack of troponin in the second hour after the clinician did not see the need for cardiac testing and follow-up. Seventeen of the patients had more than one visit with complaints of chest pain during the study period. The first applications of these patients were taken into consideration, while the other applications were excluded from the study. Two patients who developed cardiac arrest during ED evaluation were excluded from the study. Some of the patients were diagnosed with the non-coronary disease by laboratory and imaging tests. Seven of these patients were diagnosed with pneumonia, three with pulmonary thromboembolism, two with spontaneous pneumothorax, and two with myocarditis. One patient was diagnosed with pancreatitis, pericarditis, and aortic dissection despite chest pain. A total of 17 patients were excluded from the study. After 30 days of follow-up, 52 patients were excluded from the study. The study was completed with 392 patients (Figure 1).

Of the 392 patients who completed the study, $69.6 \%(n=273)$ were male, and $30.4 \%(n=119)$ were female. The mean age of the patients was $49.6 \pm 17.4$ years (min: 18 , max: 83 ). The vital signs of the patients included in the study are shown in Table 2, and their medical histories and cardiovascular risk factors are shown in Table 3. The median time after the onset of the patients' complaints was 240 minutes (IQR1: $120 \mathrm{~min}, \mathrm{IQR3}: 900 \mathrm{~min}$ ). There were new ischemic changes in the ECG of $52(13.3 \%)$ patients. The arrival and second-hour troponin values of the patients who participated in the study were recorded and evaluated. The median

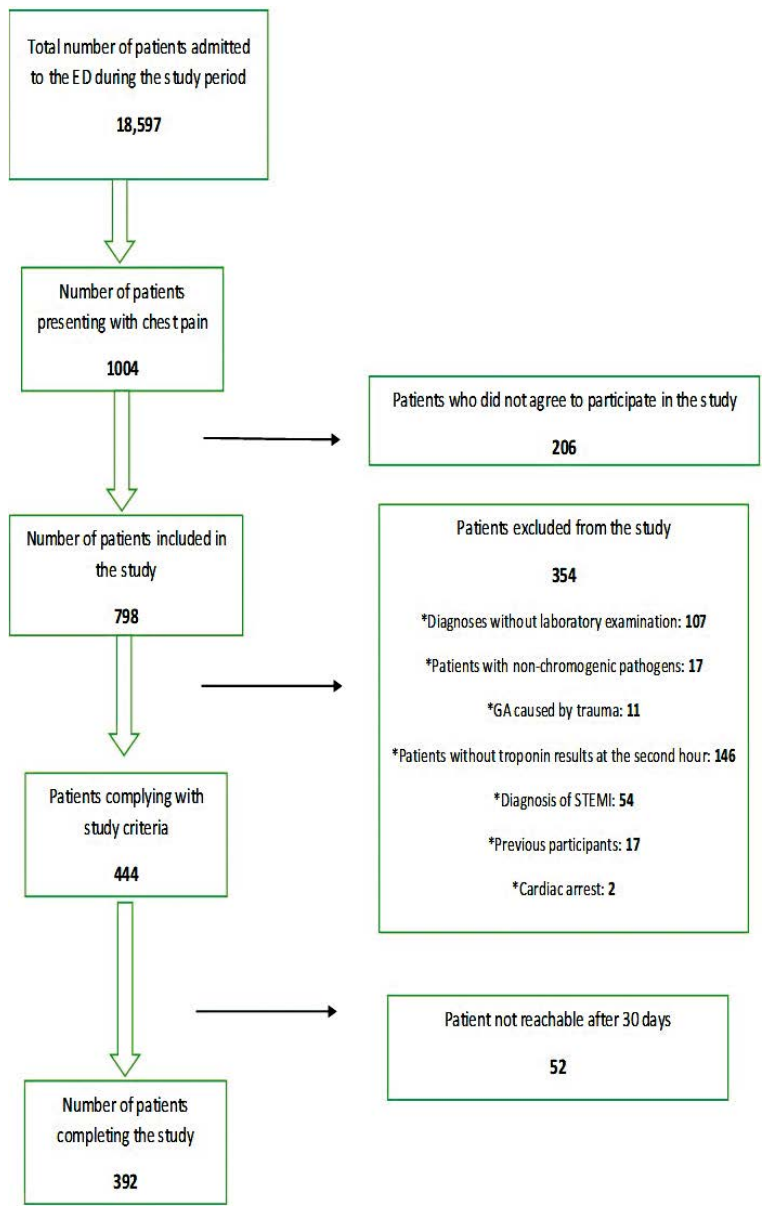

FIGURE 1. Patient flow chart

ED — Emergency Department; STEMI — ST-segment elevation myocardial infraction

Table 2. Vital findings of the patients included in
the study
\begin{tabular}{|l|c|c|c|}
\hline \multicolumn{1}{|c|}{ Vital Signs } & Average \pm SD & Minimum & Maximum \\
\hline $\begin{array}{l}\text { Systolic blood } \\
\text { pressure } \\
\text { [mm Hg] }\end{array}$ & $135.98 \pm 22.09$ & 81 & 221 \\
\hline $\begin{array}{l}\text { Diastolic blood } \\
\text { pressure } \\
\text { [mm Hg] }\end{array}$ & $80.91 \pm 14.89$ & 41 & 136 \\
\hline $\begin{array}{l}\text { Respiration } \\
\text { [breaths/min] }\end{array}$ & $16.42 \pm 3.38$ & 8 & 31 \\
\hline Temperature [ $\left.{ }^{\circ} \mathrm{C}\right]$ & $36.58 \pm 0.41$ & 35.4 & 39 \\
\hline $\begin{array}{l}\text { Fingertip oxygen } \\
\text { saturation [\%] }\end{array}$ & $95 \pm 3.41$ & 81 & 100 \\
\hline
\end{tabular}

SD — standard deviation

value of arrival troponin was $0.00 \mathrm{mcg} / \mathrm{L}$ (IQR1: 0.00 , IQR3: 0.006, minimum: 0, maximum; $16.71 \mathrm{mcg} / \mathrm{L}$, mean: $0.27 \mathrm{mcg} / \mathrm{L} \pm 1.45)$; the median value was $0.00 \mathrm{mcg} / \mathrm{L}$ (IQR1: 0.00, IQR3: 0.007, minimum: 0, maximum: 24.23 , mean value: $0.32 \mathrm{mcg} / \mathrm{L} \pm 1.80$ ). 


\begin{tabular}{|c|c|}
\hline & $\mathrm{n}(\%)$ \\
\hline Known coronary artery disease & $79(20.2 \%)$ \\
\hline COPD & $28(7.1 \%)$ \\
\hline Heart failure & $25(6.4 \%)$ \\
\hline Passed PKG & $130(33.2 \%)$ \\
\hline Passed CABG & $22(5.6 \%)$ \\
\hline Smoking & $149(38 \%)$ \\
\hline Hypertension & $102(26 \%)$ \\
\hline Dyslipidemia & $57(14.5 \%)$ \\
\hline Diabetes & $56(14.5 \%)$ \\
\hline Early coronary artery disease in the family & $54(13.8 \%)$ \\
\hline
\end{tabular}

COPD — chronic obstructive pulmonary disease; PKG — protein kinase G; $\mathrm{CABG}$ - coronary artery bypass graft; $\mathrm{n}$ - number

\section{Table 4. Distribution of patients with MACE}

\begin{tabular}{|l|c|}
\hline \multicolumn{1}{|c|}{ MACE Reason } & Patient No. (\%) \\
\hline PCI (No MI) & $8(12.3 \%)$ \\
\hline NSTEMI & $13(20 \%)$ \\
\hline NSTEMI, PCI & $37(56.9 \%)$ \\
\hline NSTEMI, PCl, CABG & $4(6.2 \%)$ \\
\hline NSTEMI, PCl, CABG, high-grade AV block & $3(4.6 \%)$ \\
\hline Total & $65(100 \%)$ \\
\hline
\end{tabular}

MACE - major adverse cardiac event; $\mathrm{PCl}$ - percutaneous coronary intervention; $\mathrm{Ml}$ - myocardial infarction; NSTEMI — non-ST-segment elevation myocardial infraction; $\mathrm{CABG}$ - coronary artery bypass graft; $\mathrm{AV}$ — atrioventricular

NSTEMI was diagnosed in $14.3 \%(n=56)$ of the patients, USAP in $8.9 \%(n=35)$, and nonspecific chest pain in $76.8 \%(n=301)$.

A total of 116 MACEs developed in 65 (16.6\%) of all patients included in the study during a onemonth follow-up. Of these 65 patients, 8 (12.3\%) underwent $\mathrm{PCl}$ without troponin elevation (without MI). In 13 (20\%), MACE was caused by NSTEMI alone, and no interventional procedures were performed. NSTEMI and PCI were associated with 37 (56.9\%) patients. NSTEMI, PCl, and CABG were observed in $4(6.2 \%)$ patients, and NSTEMI, PCI, and high-grade atrioventricular (AV) block were observed in the remaining 3 patients (4.6\%). The distribution of patients according to MACE causes is shown in Table 4. NSTEMIs included in the MACE group were diagnosed in the ED. No patients developed $\mathrm{Ml}$ after discharge. Of the patients who developed MACE, 59 were admitted to the cardiology clinic in the first ED. In 6 patients, MACE

\begin{tabular}{|c|c|c|}
\hline Clinical features & Score & $\mathrm{n}(\%)$ \\
\hline Age & & \\
\hline $18-45$ & 2 & $175(44.6 \%)$ \\
\hline $46-50$ & 4 & $27(6.9 \%)$ \\
\hline $51-55$ & 6 & $36(9.2 \%)$ \\
\hline $56-60$ & 8 & $36(9.2 \%)$ \\
\hline $61-65$ & 10 & 39 (9.9\%) \\
\hline $66-70$ & 12 & $25(6.4 \%)$ \\
\hline 71-75 & 14 & 23 (5.9\%) \\
\hline $76-80$ & 16 & $15(3.8 \%)$ \\
\hline $81-85$ & 18 & 14 (3.6\%) \\
\hline $86+$ & 20 & $2(0.5 \%)$ \\
\hline Male gender & 6 & 273 (69.6\%) \\
\hline $\begin{array}{l}\text { From } 18-50 \text { years old and } \\
\text { I. Known coronary artery disease } \\
\text { or } \\
\text { II. More than three risk factors }\end{array}$ & 4 & $29(7.4 \%)$ \\
\hline \multicolumn{3}{|l|}{ Symptoms and findings } \\
\hline Sweating & 3 & $130(33.2 \%)$ \\
\hline Pain radiates to the arm or shoulder & 5 & $181(46.2 \%)$ \\
\hline $\begin{array}{l}\text { Pain occurred or worsened by } \\
\text { inspiration }\end{array}$ & -4 & $105(26.8 \%)$ \\
\hline Pain reproduced by palpation & -6 & $52(13.3 \%)$ \\
\hline \multicolumn{3}{|l|}{ EDACS } \\
\hline$<16$ & & $251(64 \%)$ \\
\hline$>=16$ & & $141(36 \%)$ \\
\hline
\end{tabular}

EDACS - Emergency Department Assessment of Chest Pain Score-Accelerated Diagnostic Protocol; $\mathrm{n}$ - number

developed after discharge from the ED. While $81.5 \%$ of MACE patients were men, only $18.5 \%$ were women. This difference was statistically significant $(p=0.022<0.05)$. The mean age of the patients who did not develop MACE was $46.78 \pm 16.98$, and the mean age of patients who did develop MACE was $63.93 \pm 11.95$ years, and this value was statistically significant $(p=0.000<0.05)$.

Of the patients included in the study, $64 \%$ ( $n=251)$ had an EDACS of $<16$, while $36 \%$ $(n=141)$ had an EDACS $>=16$. Further, $54.3 \%$ $(n=213)$ of the patients were classified as low risk according to the EDACS-ADP, and $45.7 \%(n=179)$ were high risk. The frequencies of the EDACS determinants are shown in Table 5. MACE developed in $0.9 \%(n=2)$ of the low-risk patients, while MACE developed in $35.2 \%(n=63)$ of the high-risk patients $(p=0.000)$. According to these results, the sensitivity, specificity, PPV, NPV, + LR, and $-L R$ values of the EDACS-ADP for the thirty day evaluation of MACE in patients who presented to the study clinic with chest pain for two months were as fol- 
lows: $96.9 \%$ (95\% Cl 89,3-99.6\%), 64.5\% (95\% Cl $59.1-69,7 \%), \quad 35,2 \% \quad(95 \% \quad C l \quad 28,2-42,7 \%)$, $99,1 \%(95 \%$ Cl 96.6-99.9\%), +LR 2,73 (95\% Cl 2.35-3.18), and -LR 0,05 (95\% Cl 0.01-0.19).

\section{DISCUSSION}

To the best of the authors' knowledge, this is the first study conducted in Turkey on the EDACS-ADP system and one of the very few prospective studies on the EDACS-ADP in the world. Although there is no accepted consensus among clinicians on the rates of missed cases of MACE, many people think that a successful accelerated discharge protocol (ADP) should result in a MACE value of $<1 \%$ in patients diagnosed as low risk. This performance would correspond to an NPV of $99 \%[11,14]$. In the presented study, the rate of missed cases among low-risk patients was $0.9 \%$ and the NPV value of the whole study population was $99.1 \%$. In this regard, the use of the EDACS-ADP scoring system provides highly acceptable results in the population in which the authors are working. The rate of development of MACE was $16.6 \%$ in the study patient group. The rate was $6 \%$ in the study of Stopyra et al., $10.4 \%$ in Flaws et al., $15.4 \%$ in the derivation cohort, and $12.9 \%$ in the study of Than et al. [11-13]. Looking at MACE rates, the study patient cohort can be said to be at a higher risk, most likely because the study clinic is a part of the largest and best-equipped hospital in the area and therefore can accept more risky patients.

In the presented study, $54.3 \%$ of the patients were classified as low risk. In the EDACS-ADP derivation cohort and subsequent validation studies, this rate ranged from $41.2 \%$ to $66.7 \%$ [11-13]. In studies comparing the chest pain scores in previous emergency services, the EDACS-ADP is one of the best-rated scoring systems [15]. In the presented study, if the authors included patients with an EDACS of 12 or higher in the high-risk group, $41.3 \%(n=162)$ of all patients would be in the low-risk group. In this case, no MACE would have occurred in the low-risk group. Thus, the sensitivity of the test would reach 100\%, and its specificity would decrease to $49.5 \%$ with PPV and NPV values of $28.2 \%$ and $100 \%$, respectively. This increases the power of the test for MACE estimation and reduces the rate of missed MACEs. Even though the EDACS-ADP system decreased the EDACS score to 12 , making the EDACS-ADP more problem- atic to use for determining discharge, the EDACSADP allowed the decision to discharge more patients than many ADP tests under these conditions.

In the presented study, the authors found the EDACS-ADP concentrations to be $96.9 \%$, while in previous studies, this ratio ranged from $88.2 \%$ to $100 \%$ [11-13]. In their validation study in the U.S., Stopyra et al. [11] found this to be lower (88.2\%) than other studies and explained this as a result of the differences between the U.S. health system and that of other countries such as the more frequent use of angiography in the U.S. People in Turkey have a much higher rate of health insurance than in other parts of the world (98.6\%) [16], and as a result, physicians can easily be found to perform interventional procedures. This may explain the relatively low sensitivity value found in this study.

The troponin kit used in this study was not highly sensitive. This raises the question of whether the use of high-sensitivity troponin alone will alter the diagnostic accuracy of the existing rules. However, unlike the original derivation and validation study using high-steroidal troponin, previous studies using traditional troponin did not look at the diagnostic performance of EDACS-ADP [13].

\section{Limitations}

the study results may not be generalizable as this study was conducted in a single academic centre, for only two months, and with relatively small sample size. The main criterion for inclusion was the presence of chest pain, which would exclude some patients with atypical symptoms, such as fatigue, nausea, and dyspnea but no chest pain. In this study, the EDACS-ADP could not be used to exclude conditions such as pulmonary embolism or aortic dissection, which are rare but fatal causes of chest pain. The patient's 30-day MACE development was obtained by querying the patient or looking at the medical records, and if the medical records could not be accessed, the MACE status had to be obtained by phone. Although the assumption that the use of the EDACS-ADP system reduces hospital stays and decreases patient costs is theoretically correct, the cost analyses to prove this claim have not yet been done. Additional scientific data are needed to verify that patients have better outcomes when physicians use the EDACS-ADP method for differentiating chest pain causes than with current protocols. 


\section{CONCLUSIONS}

Most of the patients who presented to the study clinic with chest pain were classified by the EDACSADP score as low risk and suitable for discharge. The 30-day MACE rate of development was significantly low $(0.9 \%)$ in patients identified as the low-risk group and was acceptable. Therefore, this study showed that the EDACS-ADP scoring system as applied in a region different from previous study centres had comparable efficacy and safety.

\section{STATEMENTS}

Acknowledgements: The authors do not have any acknowledgements for the manuscript.

Funding: The authors declared that this study has received no financial support or any funding.

Conflict of interest: The authors declare no conflicts of interest.

Informed consent: A informed consent form from each patient who wanted to participate in the study.

Ethical approval: Ethics committee approval was received for this study from the Ethics Committee of Ataturk University Medical Faculty

(2017 - season number: 1, decision number: 40-B)

\section{REFERENCES}

1. Bruno RR, Donner-Banzhoff $N$, Söllner $W$, et al. The Interdisciplinary Management of Acute Chest Pain. Dtsch Arztebl Int. 2015; 112(45): 768-79; quiz 780, doi: 10.3238/arztebl.2015.0768, indexed in Pubmed: 26585188.

2. Almansa C, Achem S. Non-Cardiac Chest Pain of Non-Esophageal Origin. Chest Pain with Normal Coronary Arteries. 2013: 9-21, doi: 10.1007/978-1-4471-4838-8_2.

3. McCaig LF, Burt CW. National Hospital Ambulatory Medical Care Survey: 2002 emergency department summary. Adv Data. 2004(340): 1-34, indexed in Pubmed: 15068333.

4. Gerber TC, Kontos MC, Kantor B. Emergency department assessment of acute-onset chest pain: contemporary approaches and their con- sequences. Mayo Clin Proc. 2010; 85(4): 309-313, doi: 10.4065/ mcp.2010.0141, indexed in Pubmed: 20360290.

5. Kohn MA, Kwan E, Gupta M, et al. Prevalence of acute myocardial infarction and other serious diagnoses in patients presenting to an urban emergency department with chest pain. J Emerg Med. 2005; 29(4): 383-390, doi: 10.1016/j.jemermed.2005.04.010, indexed in Pubmed: 16243193.

6. Tintinalli JE, Stapczynski JS, Ma OJ, Cline DM, Meckler GD: Tintinalli's Emergency Medicine: A Comprehensive Study Guide, 8th edition: McGraw-Hill Education; 2016.

7. Ozturk TC, Guneysel 0 , Yesil 0 , et al. A New Approach To Chest Pain in the Emergency Room: "Triple Rule-Out CT. Journal of Academic Emergency Medicine. 2012; 11(1): 41-46, doi: 10.5152/jaem.2011.069.

8. Backus BE. The HEART score for chest pain patients: Utrecht University; 2012 (Dissertation) ISBN. : 9789088914195.

9. Huis In 't Veld MA, Cullen L, Mahler SA, et al. The Fast and the Furious: Low-Risk Chest Pain and the Rapid Rule-Out Protocol. West J Emerg Med. 2017; 18(3): 474-478, doi: 10.5811/westjem.2016.12.32676, indexed in Pubmed: 28435499.

10. Sanders S, Flaws $D$, Than $M$, et al. Simplification of a scoring system maintained overall accuracy but decreased the proportion classified as low risk. J Clin Epidemiol. 2016; 69: 32-39, doi: 10.1016/j.jclinepi.2015.05.006, indexed in Pubmed: 26070978.

11. Stopyra JP, Miller CD, Hiestand BC, et al. Performance of the EDACS-accelerated Diagnostic Pathway in a Cohort of US Patients with Acute Chest Pain. Crit Pathw Cardiol. 2015; 14(4): 134-138, doi: 10.1097/ HPC.0000000000000059, indexed in Pubmed: 26569652.

12. Than $M$, Flaws $D$, Sanders $S$, et al. Development and validation of the Emergency Department Assessment of Chest pain Score and $2 \mathrm{~h}$ accelerated diagnostic protocol. Emerg Med Australas. 2014; 26(1): 34-44, doi: 10.1111/1742-6723.12164, indexed in Pubmed: 24428678.

13. Flaws $D$, Than $M$, Scheuermeyer $F X$, et al. External validation of the emergency department assessment of chest pain score accelerated diagnostic pathway (EDACS-ADP). Emerg Med J. 2016; 33(9): 618-625, doi: 10.1136/emermed-2015-205028, indexed in Pubmed: 27406833.

14. Than $M$, Herbert $M$, Flaws $D$, et al. What is an acceptable risk of major adverse cardiac event in chest pain patients soon after discharge from the Emergency Department?: a clinical survey. Int J Cardiol. 2013; 166(3): 752-754, doi: 10.1016/j.ijcard.2012.09.171, indexed in Pubmed: 23084108.

15. Roche T, Jennings N, Clifford S, et al. Review article: Diagnostic accuracy of risk stratification tools for patients with chest pain in the rural emergency department: A systematic review. Emerg Med Australas. 2016; 28(5): 511 524, doi: 10.1111/1742-6723.12622, indexed in Pubmed: 27469348.

16. SGK. Genel Sağlik Sigortasi Sistemi Nisan 2017. 